\title{
Diagnosis and treatment of early lung cancer
}

Rachel McLellan, Henry Marshall, Annette Dent, Rayleen $\vee$ Bowman, Ian A Yang, Kwun M Fong

\section{Background}

Lung cancer is the leading cause of cancer death in Australia. Recently there have been unparalleled advances in the screening and management of lung cancer.

\section{Objective}

The aim of this article is to discuss diagnosis and management of lung cancer, including advances that are likely to translate into future practice.

\section{Discussion}

Screening with low-dose computed tomography scans has proven to be effective for detecting early curable disease, reducing mortality by $\geq 20 \%$ in randomised controlled trials. Implementation trials are underway within Australia and overseas, and a Commonwealth Inquiry is ongoing. Breath and blood biomarkers are less invasive alternatives that show potential but remain under investigation. Early diagnosis of lung cancer is key to improving survival - this includes familiarity with nodule screening recommendations and facilitating access to early tissue diagnosis via transthoracic needle aspiration or bronchoscopy. Treatment decisions can then be guided by staging with scans, molecular testing and multidisciplinary team consideration in the frame of patient factors/preferences. The therapeutic armamentarium is boosted by an increasing range of effective therapies including modern surgical and radiation techniques, and systemic treatments including targeted therapies and immunotherapy.
LUNG CANCER is the leading cause of cancer death in Australia, accounting for 8466 deaths in 2015 , and $5.3 \%$ of deaths overall. It causes more deaths than breast or prostate cancer, although it is diagnosed less frequently. ${ }^{1}$ The five-year survival rate for lung cancer remains extremely low at $15.8 \%,{ }^{2}$ which is attributable to the delayed emergence of symptoms and resulting late stage at diagnosis. ${ }^{3}$

Nonetheless, there have been unparalleled advances in the management of advanced lung cancer enabling meaningful prolongation of survival. These include targeted therapies directed at actionable somatic tumour mutations as well as immune checkpoint inhibitors that reactivate the host's immune response against the cancer. Additionally, prevention and early diagnosis coupled with curative treatments are key strategies for reducing lung cancer mortality by avoiding the sequelae of metastatic disease.

This brief update discusses early lung cancer, concentrating on diagnosis, which is key for enabling successful curative treatments. These now include video-assisted thoracoscopic surgery, conventional and stereotactic ablative radiotherapy, as well as combined chemoradiation/adjuvant immunotherapy for locally advanced disease. Therefore, the main focus of the article is on priority advances in screening and early diagnosis that are likely to translate to practice.

\section{Screening}

Some cancers, such as breast cancer, have much better outcomes than lung cancer. This is at least in part due to early diagnosis or early-stage curable disease, contributed to by an effective national screening program. Although screening by chest X-rays has been definitively shown in randomised controlled trials (RCTs) not to reduce lung cancer mortality, ${ }^{4}$ the advent of high-quality low-dose computed tomography (LDCT) screening is amassing increasing evidence for potential change to practice through policy.

In 2011, the National Lung Cancer Screening Trial was published, comparing annual chest X-rays to LDCT for lung cancer screening. The study investigated 53,454 people at high risk for lung cancer with annual LDCT over three years. 'High risk' was defined by the following criteria: age $55-74$ years, $\geq 30$ pack-year smoking history and being a current or ex-smoker within the past 15 years. Results showed a $20 \%$ relative reduction in mortality from lung cancer deaths with LDCT. The rate of positive LDCT results was $24.2 \%$, with $96.4 \%$ ultimately being false positives. Of patients with a positive LDCT result, $58.9 \%$ went on to have some form of clinical procedure. Complications postdiagnostic procedure occurred at a rate of $1.4 \%$ and were more likely to occur if lung cancer was present $(11.2 \%$, compared with $0.06 \%$ ). The average effective dose of LDCT was $1.5 \mathrm{mSV}$, compared with $8 \mathrm{mSv}$ for a diagnostic computed tomography (CT) scan of the chest. ${ }^{5}$ Subsequently, lung cancer screening has been recommended by the United States Preventive Services Task Force in their 2014 recommendation statement and implemented in the USA. ${ }^{6}$ 
Confirmation of the effectiveness of CT screening came from the recent NELSON RCT conference report of reduced lung cancer deaths at 10 years by $26 \%$ in men and an even larger percentage in women. ${ }^{7}$ The 2019 MILD trial also suggested a persistent benefit in reduction of mortality with prolonged screening beyond five years. ${ }^{8}$

In Australia, it has been shown that that high-quality LDCT screening is feasible and that long-term smoking cessation in a CT screening population is effective; the costs of screening and associated impacts on quality of life have also been reported. ${ }^{9-11}$ The International Lung Screen Trial is currently underway within Australia to assess the benefits of risk prediction models for patient selection to improve the efficiency of CT screening. A 2013 Australian survey of 90 patients at risk of lung cancer found the majority would be willing to participate in screening with LDCT. $^{12}$

Lung cancer screening is not currently recommended by the Australian Standing Committee on Screening; however, with mounting evidence, Federal Health Minister Hunt has called for an inquiry. ${ }^{13}$ Discussion is expected to note the integral role of smoking cessation in any screening program, and the unique potential of $\mathrm{CT}$ screening (unlike screening for other cancers) to detect important and treatable diseases including ischaemic coronary artery calcification, emphysema and osteoporosis.

\section{Pathways to diagnose suspected lung cancers}

The two main principles of lung cancer diagnosis are tissue diagnosis and staging. Tissue biopsy is required to confirm lung cancer diagnosis, determine the subtype (eg small cell, squamous cell, adenocarcinoma) and provide material for further testing for targetable tumour mutations (such as EGFR, ALK, ROS-1) and programmed death-ligand 1 (PD-L1) expression. Staging is commonly accomplished using a CT scan and positron emission tomography/CT scan, with biopsy of thoracic lymph nodes or sites of distant metastasis to confirm tumour involvement if needed. Accurate diagnosis and staging are prerequisites for identifying early curable disease and guide treatment options for advanced lung cancer. This diagnostic approach is illustrated in Figure 1. Early curable lung cancers are typically small nodules with limited nodal involvement. Methods of biopsy for these nodules include bronchoscopy and transthoracic needle aspiration (TTNA). Developments in classic fibreoptic bronchoscopy including navigation bronchoscopy and endobronchial ultrasonography (EBUS) to biopsy peripheral lesions have changed practice, enabling diagnostic biopsies in people for whom the alternative procedure of TTNA may be contraindicated. While modestly more accurate, TTNA biopsy has higher complication rates than bronchoscopy; major complication rates (pneumothorax requiring intervention, haemothorax, air embolism, needle tract seeding, and death) are approximately $5 \%$, compared with $<1 \%$ for EBUS. ${ }^{14-18}$

\section{Managing lung nodules}

As a result of the increasing use of CT technology, a greater number of nodules are encountered in clinical practice. Most of these will be benign, but the challenge is determining how best to manage these nodules in order to diagnose early lung cancers without subjecting those with benign nodules to unnecessary investigations, each with potential harms and benefits. Although there are no specific Australian guidelines, a number of high-quality guidelines are readily available and commonly used; The Fleischner Society, ${ }^{19}$ British Thoracic Society, ${ }^{20}$ American College of Chest Physicians $^{21}$ and Cancer Council Australia (www.cancer.org.au/health-professionals/ clinical-guidelines/lung-cancer.html) have produced evidence-based nodule and cancer guidelines (Table 1). The guidelines are important tools that can reduce healthcare variation and patient harm, and improve patient outcomes and communication. The guidelines suggest risk assessment of nodule and patient (eg nodule size, location; patient age, smoking history, cancer history, etc) and review of historical CT scans to ascertain stability. One emerging challenge for the adoption of these guidelines in Australia is the increasing reliance on volumetrics for threshold assessment and for sensitive identification of short-term growth as a marker for malignant potential, which is not routine clinical practice.

The implication of these technological advances is that it is now much easier and safer to accurately diagnose and stage patients presenting with suspected lung cancer, thus avoiding the risks of empirical treatment of suspected but pathologically unconfirmed lung cancer. However, they do not have a first-line role in the screening of asymptomatic individuals, which has led to efforts to develop non-invasive tests for lung cancer.

\section{Biomarkers}

There has been a surge of interest in lung cancer biomarkers in recent years as researchers evaluate biological specimens such as sputum, saliva, buccal swabs, breath condensate and breath volatile organic compounds (VOCs). Thousands of resulting publications describe these potential lung cancer biomarker candidates. These minimally invasive tests are relatively safe, acceptable to consumers and can be repeated more frequently than tissue biopsy, which for some people with lung cancer is not feasible or too high risk because of comorbidities.

\section{Breath biomarkers}

Analysis of exhaled breath for VOCs is a new approach to the diagnosis of lung cancer. As early as Roman times, the smell of a person's breath has assisted physicians with the diagnosis of a disease; for example, uncontrolled diabetes was associated with a sweet, acetone odour; ${ }^{22}$ liver failure produced a fish-like smell, ${ }^{23}$ and renal failure was identified by a urine-like smell. ${ }^{24} \mathrm{McCulloch}$ showed that dogs could be trained to detect lung cancer and breast cancer in people with various stages of disease with almost $100 \%$ accuracy, merely by smelling the subject's breath. ${ }^{25}$ These observations suggest that there are biomarkers in exhaled breath that are potentially useful for diagnosing 
Table 1. Relevant Australian and international guidelines on pulmonary nodule surveillance and pathways for lung cancer diagnosis that may be of use for Australian general practitioners

Australian guidelines

Cancer Council Australia

Guidelines outlining optimal care for lung cancer by stage including stepwise practice advice on timelines, investigations and management. Two-page summary available.

- Optimal care pathways for people with lung cancer, www.cancer.org.au/health-professionals/optimal-cancer-carepathways.html

- Optimal care pathways for people with lung cancer: Quick reference guide, www.cancer.org.au/content/ocp/quick/Lung_ cancer_OCP_Quick_reference_guide.PDF

\section{Cancer Australia}

International guidelines

Fleischner Society

Guidelines on incidentally detected pulmonary nodule follow up. Includes photographic demonstration of nodule characteristics.

- Guidelines for management of incidental pulmonary nodules detected on CT images: From the Fleischner Society 2017, https://pubs.rsna.org/doi/full/10.1148/radiol.2017161659

British Thoracic Society

A two-page practical summary to guide general practitioners on risk factors and investigating symptoms of lung cancer.

- Investigating symptoms of lung cancer: A guide for GPS, https://canceraustralia.gov.au/publications-and-resources/ cancer-australia-publications/investigating-symptoms-lungcancer-guide-gps

Summaries of the optimal framework for quality lung cancer care for professionals and consumers.

- Delivering best practice lung cancer care - A summary for health professionals, https://canceraustralia.gov.au/sites/ default/files/publications/lung-cancer-framework-resourceshealth-professional-summary/pdf/lung_cancer_-_framework_ resources_-_health_professional_summary.pdf

- Getting the best care and advice - A guide for those affected by lung cancer, https://canceraustralia.gov.au/sites/default/files/ publications/lung-cancer-framework-resources-consumersummary/pdf/lung_cancer__framework_resources_-_ consumer_summary.pdf

\section{Guidelines on initial and follow-up approach for pulmonary} nodules as well as detailed evidence-based recommendations for diagnosis and management of lung cancer updated in March 2019.

- British Thoracic Society guidelines for the investigation and management of pulmonary nodules, https://thorax.bmj.com/ content/thoraxjnl/70/Suppl_2/ii1.full.pdf

- Lung cancer: Diagnosis and management, www.nice.org.uk/ guidance/ng122

American College
of Chest Physicians
(ACCP)

Detailed summary of pulmonary nodule follow-up and diagnosis and management of lung cancer. Includes table of investigation harm and benefits.

- Evaluation of individuals with pulmonary nodules: When is it lung cancer?, www.ncbi.nlm.nih.gov/pmc/articles/ PMC3749714/pdf/chest_143_5_suppl_e93S.pdf

- Executive summary, https://journal.chestnet.org/article/ S0012-3692(13)60283-4/pdf disease. Studies examining exhaled breath using gas chromatography and mass spectrometers have identified individual chemical compounds associated with lung cancer and confirmed that there is not one single VOC, but rather a combination of VOCs. ${ }^{26}$ However, these techniques have limited applicability in the clinical setting because of their expense and difficulty of use, and the need for highly experienced analysts to operate and interpret the results. Electronic noses and related instruments are simpler, cheaper and easier to use, facilitating their utilisation in the clinical setting. These instruments employ different sensor array technologies to identify VOC patterns or smellprints rather than individual compounds. Studies using a variety of these instruments, different sampling techniques and different statistical analyses have consistently discriminated between groups of subjects with lung cancer and control subjects. ${ }^{27}$ Small studies have shown a sensitivity of the exhaled breath VOC test between $73 \%$ and $100 \%$, and specificity between $48 \%$ and $100 \%$. Therefore, further research needs to be performed before it can become a clinical tool. Large-scale multicentre clinical trials and validation in independent at-risk subjects are required to improve the clinical sensitivity and specificity of the test. ${ }^{28}$

\section{Blood biomarkers}

Liquid biopsy aims to detect and quantify circulating cancer biomarkers by analysing biological fluids, predominantly blood samples. These biomarkers contain abundant genomic, proteomic and metabolomic information that can be used for early disease detection (diagnostic biomarkers) and to predict treatment response and relapse (prognostic/ predictive biomarkers). Biomarkers include tumour-associated antigens, tumourassociated autoantibodies, circulating tumour cells, circulating tumour DNA (ctDNA), microRNA and exosomes.

Early lung cancer with low-volume disease has intrinsic challenges for platforms that require demonstration of direct tumour attributes (such as somatic tumour DNA mutations), as the low amounts of ctDNA in blood in early lung 
cancer relative to late-stage cancer are below the diagnostic limit of detection (even with the current next-generation sequencing platforms). Some biomarkers may be more effective when used in combination with traditional serum cancer markers such as the multi-analyte CancerSEEK (ctDNA and protein) test. Although some biomarkers have progressed to RCTs in clinical populations, ${ }^{29-32}$ none has achieved sufficient levels of analytical/ clinical validity and clinical utility to enter mainstream use.

\section{Key implications}

Anti-cancer therapeutics are dramatically changing outcomes of metastatic lung cancer. They are benefitting a subset of responders but are counterbalanced by a different spectrum of treatment toxicities including financial ones. Earlier in the natural history, prevention and screening for curable disease can help avoid metastatic disease. Lung cancer screening with CT provides a $20 \%$ or greater reduction in lung cancer mortality. It has already been implemented overseas and is currently under investigation in Australia. Nodules are a very common incidental finding that should be managed in line with international and/or national guidelines; it is important that healthcare providers familiarise themselves with these to avoid over-investigation and under-diagnosis of cancer. In the meantime, important national resources are available to assist with achieving best practice and outcomes for people at risk of and with lung cancers. Namely, use of the Australian Optimal Care Pathways, with demonstrated ability to prolong survival in colorectal cancer, and the Cancer Australia lung cancer guidance and the Lung Cancer Framework. ${ }^{33,34}$ Both emphasise timely assessment and referral of suspected lung cancer for tissue diagnosis, staging and consideration of patient preferences and comorbidities by a multidisciplinary team to achieve optimal outcomes. An active partnership between primary care physicians and their local multidisciplinary teams is key to enabling cure where possible and palliation to optimise quality of life otherwise for all affected by lung cancer.

\section{Authors}

Rachel McLellan MBBS, FRACP (Gen Med), Associate Lecturer of the School of Clinical Medicine, UQ Thoracic Research Centre, The Prince Charles Hospital, University of Queensland, Qld; Registrar, Department of Thoracic Medicine, The Prince Charles Hospital, Metro North Hospital and Health Service, Qld. racheljegerton@gmail.com

Henry Marshall MBBS, PhD, FRACP (Thor Med), Senior Lecturer and Clinical Academic Fellow, UQ Thoracic Research Centre, The Prince Charles Hospital, University of Queensland, Qld; Thoracic Physician, Department of Thoracic Medicine, The Prince Charles Hospital, Metro North Hospital and Health Service, Qld

Annette Dent BAppSc (Med Tech), PhD, Director, Respiratory Investigations, Department of Thoracic Medicine, The Prince Charles Hospital, Metro North Hospital and Health Service, Qld

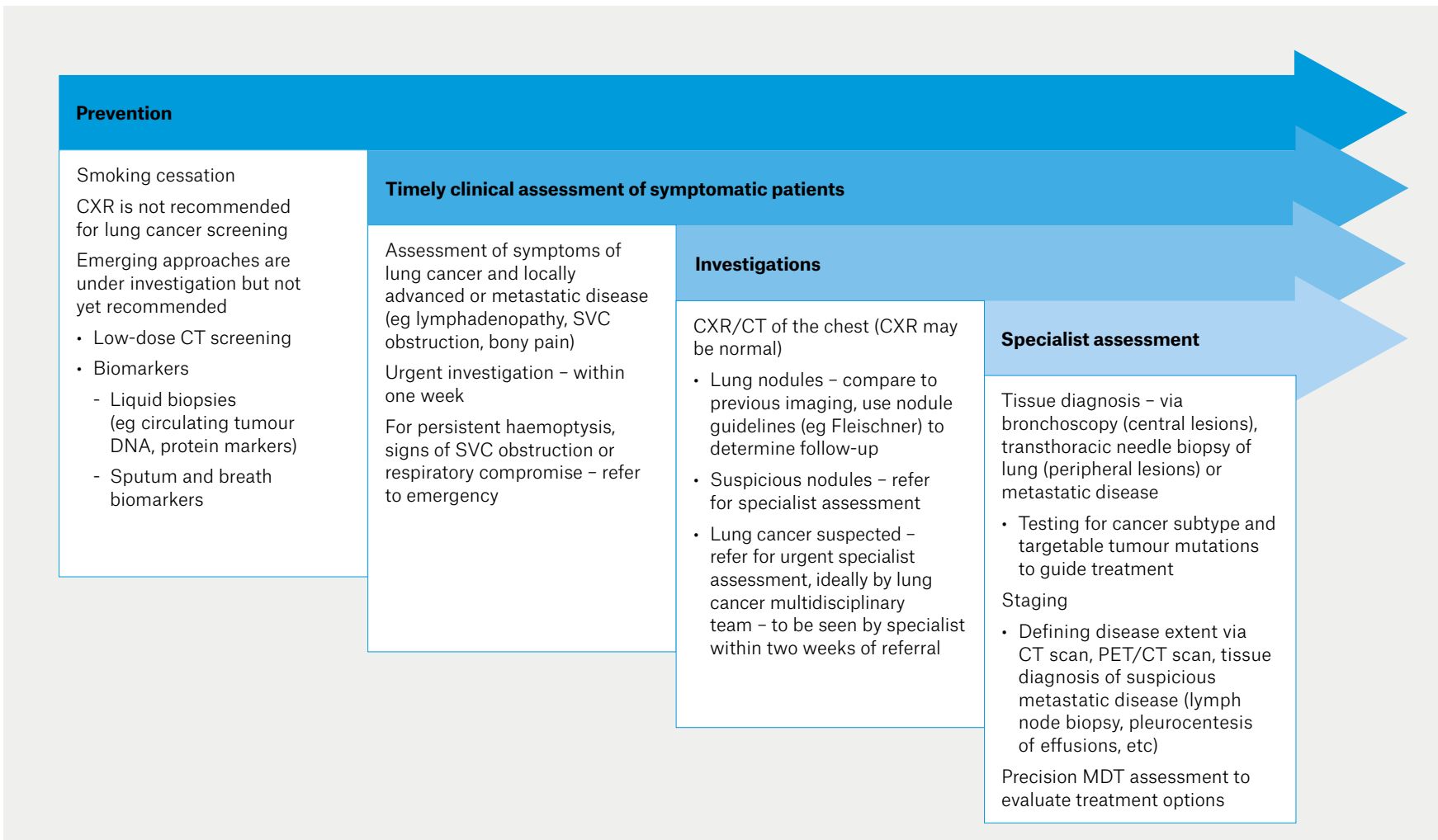

Figure 1. An approach to lung cancer diagnosis in $2020^{35}$

$C T$, computed tomography; CXR, chest X-ray; MDT, multidisciplinary team; PET, positron emission tomography; SVC, superior vena cava 
Rayleen V Bowman MBBS, PhD, FRACP (Thor Med), Associate Professor, UQ Thoracic Research Centre, The Prince Charles Hospital, University of Queensland, Qld; Thoracic Physician, Department of Thoracic Medicine, The Prince Charles Hospital, Metro North Hospital and Health Service, Qld Ian A Yang MBBS (Hons), PhD, FRACP (Thor Med), FAPSR, FThorSoc, Grad Dip Clin Epid, Professor, Medicine and Head UQ Northside Clinical Unit, UQ Thoracic Research Centre, The Prince Charles Hospital, University of Queensland, QId; Thoracic Physician and Director, Department of Thoracic Medicine, The Prince Charles Hospital, Metro North Hospital and Health Service, Qld

Kwun M Fong MBBS, PhD, FRACP (Thor Med), Professor, Medicine, UQ Thoracic Research Centre, The Prince Charles Hospital, University of Queensland, Qld; Thoracic Physician and Head of Pulmonary Malignancy Services, Department of Thoracic Medicine, The Prince Charles Hospital Metro North Hospital and Health Service, Qld Competing interests: KMF reports grants from various competitive funding bodies, travel support for talks at industry-sponsored scientific meetings - pro bono, occasional honoraria for thesis marking for various universities, occasional honoraria for grant review for various international funding bodies, occasional royalty for review work from UpToDate, and occasional honoraria for report review for Cochrane Clinical Answers, outside the submitted work. Funding: None.

Provenance and peer review: Not commissioned, externally peer reviewed.

\section{References}

1. Australian Bureau of Statistics. Causes of death, Australia, 2015. Belconnen, ACT: ABS, 2017. Available atwww.abs.gov.au/ausstats/abs@.nsf/ Lookup/by\%20Subject/3303.0 2015 Main\%20 Features Lung\%20cancer 10004 [Accessed 4 March 2020].

2. Australian Institute of Health and Welfare. Cancer in Australia 2017. Cancer Series no. 101. Cat. no. CAN 100. Canberra, ACT: AlHW, 2017.

3. Lewis JA, Petty WJ, Tooze JA, et al. Low-dose CT lung cancer screening practices and attitudes among primary care providers at an academic medical center. Cancer Epidemiol Biomarkers Prev 2015;24(4):664-70. doi: 10.1158/1055-9965. EPI-14-1241.

4. Oken MM, Hocking WG, Kvale PA, Andriole GL, Buys SS, Church TR, et al. Screening by chest radiograph and lung cancer mortality: The Prostate, Lung, Colorectal, and Ovarian (PLCO) randomized trial. JAMA 2011;306(17):1865-73. doi: 10.1001/jama.2011.1591.

5. Aberle DR, Adams AM, Berg CD, et al. Reduced lung-cancer mortality with low-dose computed tomographic screening. N Engl J Med 2011;365(5):395-409. doi: 10.1056/ NEJMoa1102873.

6. Moyer VA. Screening for lung cancer: U.S. Preventive Services Task Force recommendation statement. Ann Intern Med 2014;160(5):330-38. doi: 10.7326/M13-2771.

7. de Koning $\mathrm{HJ}$, van der Aalst $\mathrm{CM}$, de Jong PA, et al. Reduced lung-cancer mortality with volume CT screening in a randomized trial. N Engl J Med 2020;382:503-13. doi: 10.1056/NEJMoa1911793.

8. Pastorino U, Silva M, Sestini S, et al. Prolonged lung cancer screening reduced 10-year mortality in the MILD trial: New confirmation of lung cancer screening efficacy. Ann Oncol 2019;30(7):1162-69. doi: 10.1093/annonc/mdz117.
9. Marshall HM, Bowman RV, Ayres J, et al. Lung cancer screening feasibility in Australia. Eur Respir J 2015;45(6):1734-37. doi: 10.1183/09031936.00208714.

10. Marshall HM, Finn N, Bowman RV, et al. Cost of screening for lung cancer in Australia. Intern Med J 2019;49(11):1392-99. doi: 10.1111/imj.14439.

11. Vemula M, Marshall HM, Yang IA, Bowman RV, Fong KM. Active screening for lung cancer increases smoking abstinence -5 year observational data from the Queensland Lung Cancer Screening Study (QLCSS). The Australia \& New Zealand Society of Respiratory Science and The Thoracic Society of Australia and New Zealand (ANZSRS/TSANZ) Annual Scientific Meeting; 23-27 March 2018; Adelaide, Australia. Richmond, VIC: Wiley-Blackwell Publishing Asia doi: 10.1111/resp.13268.

12. Flynn AE, Peters MJ, Morgan LC. Attitudes towards lung cancer screening in an Australian high-risk population. Lung Cancer Int 2013;2013:789057. doi: 10.1155/2013/789057.

13. Hunt G. Building a mentally and physically healthy Australia 2019. Canberra, ACT: Ministers Department of Health, 2019. Available at www. health.gov.au/ministers/the-hon-greg-hunt-mp/ media/building-a-mentally-and-physicallyhealthy-australia [Accessed 3 March 2020].

14. Heerink WJ, de Bock GH, de Jonge GJ, Groen HJM, Vliegenthart R, Oudkerk M. Complication rates of CT-guided transthoracic lung biopsy: Meta-analysis. Eur Radiol 2017;27(1):138-48. doi: 10.1007/s00330-016-4357-8.

15. Ali MS, Trick W, Mba BI, Mohananey D, Sethi J, Musani Al. Radial endobronchial ultrasound for the diagnosis of peripheral pulmonary lesions: $A$ systematic review and meta-analysis. Respirology 2017;22(3):443-53. doi: 10.1111/resp.12980.

16. Han Y, Kim HJ, Kong KA, et al. Diagnosis of small pulmonary lesions by transbronchial lung biopsy with radial endobronchial ultrasound and virtual bronchoscopic navigation versus CT-guided transthoracic needle biopsy: A systematic review and meta-analysis. PLoS One 2018;13(1):e0191590. doi: 10.1371/journal.pone.0191590.

17. Verdial FC, Berfield KS, Wood DE, et al. Safety and costs of endobronchial ultrasound-guided nodal aspiration and mediastinoscopy. Chest 2019 doi: 10.1016/j.chest.2019.09.021.

18. Steinfort DP, Khor YH, Manser RL, Irving LB. Radial probe endobronchial ultrasound for the diagnosis of peripheral lung cancer: Systematic review and metaanalysis. Eur Respir J 2011;37(4):902-10. doi: 10.1183/09031936.00075310.

19. MacMahon $H$, Naidich DP, Goo JM, et al. Guidelines for management of incidental pulmonary nodules detected on CT images: From the Fleischner Society 2017 Radiology 2017;284(1):228-43. doi: 10.1148/ radiol.2017161659.

20. Callister MEJ, Baldwin DR, Akram AR, et al. British Thoracic Society guidelines for the investigation and management of pulmonary nodules. Thorax 2015;70(Suppl 2):ii1-ii54. doi: 10.1136/ thoraxjnl-2015-207168.

21. Gould MK, Donington J, Lynch WR, et al. Evaluation of individuals with pulmonary nodules: When is it lung cancer? Diagnosis and management of lung cancer. 3rd edn. American College of Chest Physicians evidence-based clinical practice guidelines. Chest 2013;143(5 Suppl):e93S-120S. doi: 10.1378/chest.12-2351.
22. Rollo J. Cases of the diabetes mellitis: With the results of the trials of certain acids and other substances in the cure of lues venerea. London: C. Dilly, Poultry, 1798.

23. Humbert JR, Hammond KB, Hathaway WE, Marcoux JG, O'Brien D. Trimethylaminuria: The fish-odour syndrome. Lancet 1970;296(7676):770-71. doi: 10.1016/S01406736(70)90241-2.

24. Buszewski B, Kesy M, Ligor T, Amann A. Human exhaled air analytics: Biomarkers of diseases. Biomed Chromatogr 2007;21(6):553-66. doi: $10.1002 /$ bmc.835.

25. McCulloch M, Jezierski T, Broffman M, Hubbard A, Turner K, Janecki T. Diagnostic accuracy of canine scent detection in early- and late-stage lung and breast cancers. Integr Cancer Ther 2006;5(1):30-39. doi: 10.1177/1534735405285096.

26. Jia Z, Patra A, Kutty VK, Venkatesan T. Critical review of volatile organic compound analysis in breath and in vitro cell culture for detection of lung cancer. Metabolites 2019;9(3). doi: 10.3390/ metabo9030052.

27. Bikov A, Lázár Z, Horvath I. Established methodological issues in electronic nose research: How far are we from using these instruments in clinical settings of breath analysis? J Breath Res 2015;9(3):034001. doi: 10.1088/17527155/9/3/034001.

28. Einoch Amor R, Nakhleh MK, Barash O, Haick $\mathrm{H}$. Breath analysis of cancer in the present and the future. Eur Respir Rev 2019;28(152). doi: 10.1183/16000617.0002-2019.

29. Calabrese F, Lunardi F, Pezzuto F, et al. Are there new biomarkers in tissue and liquid biopsies for the early detection of non-small cell lung cancer? J Clin Med 2019;8(3):414. doi: 10.3390/ jcm8030414.

30. Liang W, Zhao Y, Huang W, Liang $\mathrm{H}$, Zeng $\mathrm{H}$, He J. Liquid biopsy for early stage lung cancer. J Thorac Dis 2018;10(Suppl 7):S876-S81. doi: 10.21037/ jtd.2018.04.26.

31. Hanash SM, Ostrin EJ, Fahrmann JF. Blood based biomarkers beyond genomics for lung cancer screening. Transl Lung Cancer Res 2018;7(3):327-35. doi: 10.21037/tlcr.2018.05.13.

32. Sullivan FM, Farmer E, Mair FS, et al. Detection in blood of autoantibodies to tumour antigens as a case-finding method in lung cancer using the EarlyCDT(R)-Lung Test (ECLS): Study protocol for a randomized controlled trial. BMC Cancer 2017;17(1):187. doi: 10.1186/s12885-017-3175-y.

33. Cancer Australia. Investigating symptoms of lung cancer: A guide for GPs. Surry Hills, NSW: Cancer Australia, 2012.

34. te Marvelde L, McNair P, Whitfield K, et al. Alignment with indices of a care pathway is associated with improved survival: An observational population-based study in colon cancer patients. E Clinical Medicine 2019;15:42-50. doi: 10.1016/j. eclinm.2019.08.009.

35. Cancer Council. Optimal care pathway for people with lung cancer. Sydney, NSW: Cancer Council, 2016. 